\title{
On Optimizing Sensor Placement for Spatio-Temporal Temperature Estimation in Large Battery Packs
}

\author{
Philipp Wolf ${ }^{1}$, Scott Moura ${ }^{2}$ and Miroslav Krstic ${ }^{2}$
}

\begin{abstract}
This paper optimizes the number of sensors and their locations for estimating the 2-D spatio-temporal temperature dynamics in large battery packs. Monitoring temperature in battery packs is crucial for safety, efficiency, and longterm endurance. The temperature dynamics in large battery packs evolve in space and time, whereas sensors only provide pointwise data. Moreover, the number of sensors should be minimized to reduce costs. The temperature dynamics are modeled by a system of linear two-dimensional heat partial differential equations (PDEs). In this paper we perform eigendecomposition of the PDEs to produce a finite-dimensional model. Modal observability is defined from the magnitude of these eigenmodes. The process of optimizing the number and location of sensors involves two steps: First, a binary optimization minimizes the number of sensors. Second, a constrained nonlinear programming problem is solved to optimize the previously found sets with respect to a min-max-type objective function. The optimization procedure is independent of the estimator design.
\end{abstract}

\section{INTRODUCTION}

This paper presents a systematic method to optimally place a minimally-sized sensor set for estimation of battery pack thermal dynamics.

Monitoring and controlling battery pack temperature dynamics is a critically important problem. Large-scale devices, such as electric vehicles and grid-scale energy storage, require high energy and power. Consequently, battery packs are often constructed with thousands of cells. One important challenge with such battery packs is that an individual cell may become thermally unstable. The heat generated from this unstable cell can transfer to neighboring cells, thereby causing a chain reaction commonly called thermal runaway. Even when cells do not enter this unstable region, high temperatures can accelerate degradation mechanisms and low temperatures limit available power/energy [1]. Consequently, accurately monitoring and regulating battery pack temperature is a crucial component of battery management systems [2], [3], [4]. CFD modeling, model reduction, and analysis of battery pack thermal dynamics is currently an active research area [5], [6], [7], [8]. Intelligent sensor placement is the next step towards advanced thermal management. Nonetheless, sensor placement in distributed parameter systems bears several challenges. First, placing multiple sensors is NP-

This work was supported by the German Academic Exchange Service (DAAD) and the National Science Foundation

${ }^{1} \mathrm{P}$. Wolf is with the Institute for System Dynamics, Faculty 7, University of Stuttgart, 70550 Stuttgart, Germany

${ }^{2} \mathrm{~S}$. Moura and M. Krstic are with the Department of Mechanical and Aerospace Engineering, University of California, San Diego, CA 92093, USA (email: smoura@ucsd.edu; krstic@ucsd.edu) hard [9]. The current work addresses this issue. Second, the optimization problem is non-convex, in general.

The engineering literature on optimal sensor placement examines various optimization criteria. These can be classified into open-loop and closed-loop considerations. Open-loop methods often focus on observability metrics, e.g. the spatial $\mathcal{H}_{2}$ norm [10], [11]. Closed-loop methods simultaneously design the observer gains and sensor locations [12], [13], and consider metrics such as estimation error regulation, disturbance rejection, and robustness [14]. The survey paper [15] provides an excellent analysis of various aspects of actuator/sensor placement.

The current work focuses on placement of a minimally sized sensor set, with respect to a particular observability metric, inspired by eigenanalysis. We focus on a 2D PDE model for battery pack thermal dynamics recently proposed in [16]. Our main idea is to derive a finite-dimensional modal model from the aforementioned PDE model via eigenanalysis. The modal setting provides a natural definition for an observability measure we use for sensor placement. In addition, we focus on computing the minimal sensor set to achieve a given observability criterion. This problem is solved by capitalizing on a submodularity property [17], which substantially reduces computational effort. Finally, the sensor placement problem is mathematically cast as a nonlinear optimization problem.

In Section II, the modal model is derived from the PDE model. Next, a systematic method for optimal sensor placement is presented in Section III, followed by some exemplary results in Section IV. The paper's main ideas are summarized in Section V.

\section{Modelling}

We use the PDE model developed in [16]. The two spatial dimensions are denoted $x$ and $y$, with pack boundaries at $x= \pm L_{x}$ and $y= \pm L_{y}$, so that the battery pack resembles a rectangular shape within a rectangular domain

$$
\Omega=\left[-L_{x}, L_{x}\right] \times\left[-L_{y}, L_{y}\right] .
$$

Time is assumed to run from $t=0$ to $t=\infty$.

\section{A. PDE model}

The model for temperature distribution in the battery pack has three states, $\Theta, T_{h}, T_{c}$, which respectively represent temperature in the pack material, cells, and cooling channels. 


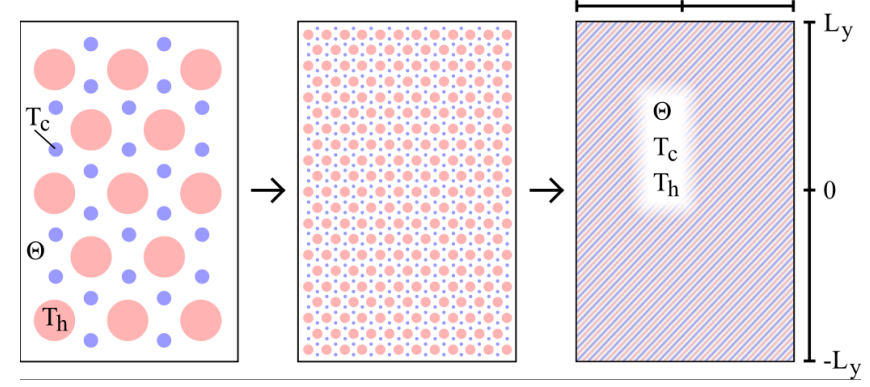

Fig. 1. Illustration of the PDE approximation for battery packs with a large number of cells. Cells are illustrated in red, cooling channels in blue, and pack material in white. Note that states $\Theta, T_{c}, T_{h}$ exist over the entire domain $\Omega$ in the final PDE model.

It is mathematically given by

$$
\begin{aligned}
\frac{\partial}{\partial t} \Theta(x, y, t)= & a \triangle \Theta(x, y, t)+b\left[T_{h}(x, y, t)-\Theta(x, y, t)\right] \\
& +c\left[T_{c}(x, y, t)-\Theta(x, y, t)\right], \\
\frac{\partial}{\partial t} T_{h}(x, y, t)= & d\left[\Theta(x, y, t)-T_{h}(x, y, t)\right]+e \Phi_{h}(x, y, t), \\
\frac{\partial}{\partial t} T_{c}(x, y, t)= & f\left[\Theta(x, y, t)-T_{c}(x, y, t)\right]-g \Phi_{c}(x, y, t),
\end{aligned}
$$

where $\triangle=\frac{\partial^{2}}{\partial x^{2}}+\frac{\partial^{2}}{\partial y^{2}}$ is the Laplacian operator,

$$
a, b, c, d, e, f, g \in \mathbb{R},
$$

are constant coefficients,

$$
\Theta(x, y, 0), \quad T_{h}(x, y, 0), \text { and } T_{c}(x, y, 0),
$$

denote the initial conditions, and

$$
\begin{aligned}
& \left.\frac{\partial}{\partial x} \Theta(x, y, t)\right|_{x= \pm L_{x}}=\left.\mp \frac{\lambda}{k_{p}} \Theta(x, y, t)\right|_{x= \pm L_{x}} \\
& \left.\frac{\partial}{\partial y} \Theta(x, y, t)\right|_{y= \pm L_{y}}=\left.\mp \frac{\lambda}{k_{p}} \Theta(x, y, t)\right|_{y= \pm L_{y}}
\end{aligned}
$$

denote the Robin-type boundary conditions. The variables $\Phi_{h}$ and $\Phi_{c}$ denote the heat generated by the cells and the heat taken by the cooling system, respectively.

The PDE model was derived in [16] by dividing the battery pack domain into subdomains, each of which contained either a cell, cooling channel, or the pack material. Then the dimensions of these subdomains were taken to zero. This represents a large number of cells (see Figure 1). Please note that we have modified the model by subtracting the ambient temperature from each state, thus maintaining the structure of the PDE system. This positions us to perform an eigenanalysis and derive the modal model, described next.

\section{B. Modal model}

We achieve the modal version of the PDEs by calculating an orthonormal basis of eigenmodes over the domain $\Omega$ and projecting the PDEs onto this basis. This process produces an infinite set of ODEs, which we summarize next (for more details please refer to [18]). Assuming $\Theta(x, y, t)=$ $\mu_{\Theta}(t) Z(x, y)$, the homogeneous part of (2) becomes

$$
\frac{\frac{d}{d t} \mu_{\Theta}}{a \mu_{\Theta}}+\frac{b+c}{a}=-2 k=\frac{\triangle Z}{Z}
$$

with the separation constant $-2 k$. We separate this into two independent ODEs governing the temporal and spatial parts of the solution as

$$
\begin{aligned}
\frac{d}{d t} \mu_{\Theta}(t) & =-(2 k a+b+c) \mu_{\Theta}(t), \\
\triangle Z(x, y) & =-2 k Z(x, y) .
\end{aligned}
$$

The solution of (10) is given by

$$
\mu_{\Theta}(t)=\mu_{\Theta}(t) e^{-(2 k a+b+c) t} .
$$

For the spatial part, we assume $Z(x, y)=X(x) Y(y)$ and (11) can be arranged as

$$
\frac{\frac{\partial^{2}}{\partial x^{2}} X}{X}+k=h=-\frac{\frac{\partial^{2}}{\partial y^{2}} Y}{Y}-k .
$$

which is separated into two Sturm-Liouville equations

$$
\begin{aligned}
X^{\prime \prime}+(k-h) X & =0, \\
Y^{\prime \prime}+(k+h) Y & =0
\end{aligned}
$$

with the boundary conditions

$$
\begin{aligned}
X^{\prime}\left( \pm L_{x}\right) & =\mp \frac{\lambda}{k_{p}} X\left( \pm L_{x}\right), \\
Y^{\prime}\left( \pm L_{y}\right) & =\mp \frac{\lambda}{k_{p}} Y\left( \pm L_{y}\right),
\end{aligned}
$$

derived from (7) and (8). The solution of (14), (16) for $X$ is an infinite set $(i \in \mathbb{N})$ of eigenfunctions

$$
X_{i}(x)= \begin{cases}\cos \left(\gamma_{x i} x\right) & \text { for odd } i \\ \sin \left(\gamma_{x i} x\right) & \text { for even } i\end{cases}
$$

where $\gamma_{x}=\sqrt{k-h}$ solves

$$
\tan \left(\gamma_{x} L_{x}\right)=\frac{\lambda}{k_{p}} \frac{1}{\gamma_{x}}
$$

for the cosine eigenfunctions and

$$
\tan \left(\gamma_{x} L_{x}\right)=-\frac{k_{p}}{\lambda} \gamma_{x}
$$

for the sine eigenfunctions. We get a structurally identical solution for $Y$ and in combination, these sets form the eigenmodes for (2), consisting of the mode shapes $Z_{i j}(x, y)=$ $X_{i}(x) Y_{j}(y), i, j=1, \ldots, \infty$ with corresponding separation constants $k_{i j}=\left(\gamma_{x i}^{2}+\gamma_{y j}^{2}\right) / 2$.

Note from (12) that the eigenvalues, given by $-\left(2 k_{i j} a+\right.$ $b+c)$, are real, negative, and increase in an approximately quadratic fashion towards $-\infty$ as the indices $i, j$ increase.

The eigenmodes are $L_{2}$-orthogonal [18], [19] and are normalized by pre-multiplying by the constant

$$
K_{i j}=\frac{1}{\sqrt{\int_{\Omega} Z_{i j}^{2} d \Omega}},
$$


forming an orthonormal basis $K_{i j} Z_{i j}, i, j=1, . ., \infty$ for (2).

Equations (3) and (4) are ODEs in time, parameterized over space. Since they are coupled with (2), their solutions inherit the same spatial structure. For this reason, and the fact that the eigenmodes provide an orthogonal basis, the whole system of PDEs is projected onto the eigenmodes.

Thus, we obtain an infinite set of systems of ODEs $(n=$ $1, \ldots, \infty)$

$$
\begin{aligned}
\frac{d}{d t} \mu_{\Theta n} & =-\left(2 a k_{n}+b+c\right) \mu_{\Theta n}+b \mu_{T_{h} n}+c \mu_{T_{c} n} \\
\frac{d}{d t} \mu_{T_{h} n} & =-d \mu_{T_{h} n}+d \mu_{\Theta n}+e K_{n} \int_{\Omega} \Phi_{h} Z_{n} d \Omega \\
\frac{d}{d t} \mu_{T_{c} n} & =-f \mu_{T_{c} n}+f \mu_{\Theta n}+g K_{n} \int_{\Omega} \Phi_{c} Z_{n} d \Omega
\end{aligned}
$$

where $n$ indexes the Cartesian product of $i=1, \ldots, \infty$ and $j=1, \ldots, \infty$. The modal coefficients $\mu_{\Theta n}, \mu_{T_{h} n}$ and $\mu_{T_{c} n}$ characterize the temporal part of the solution.

A finite-dimensional ODE model is obtained by taking a finite subset of (22) - (24), containing only the first $N$ eigenmodes. This truncation is justified by the fact that the model is stable and the eigenvalues increase approximately quadratically towards $-\infty$, as the index number increases.

\section{Sensor Palcement}

Next we investigate optimal in-domain placement of temperature sensors for a modal model-based estimator. Sensor placement, in general, has many different aspects, which include, but are not limited to observability, robustness, and noise/disturbance rejection. We choose one of these aspects to explicitly account for in our optimization: the modal observability. In view of this requirement, the task is two-fold. First, determine the minimal number of sensors. Second, place the sensors over the domain in some optimal sense. This problem is, in general, an NP-hard, constrained, mixedinteger, nonlinear program. For the approach presented here, the problem is split into two steps: First, we define a fixed minimum threshold for the observability and a grid on the domain. On the grid points, we perform a binary integer search, minimizing the number of sensors and finding sets that satisfy the minimum observability requirement. In the second step, we take all sensor sets from the first step and optimize their location, now with respect to maximizing the observability in a constrained nonlinear optimization.

\section{A. Modal observability measure}

Modal observability is a well known concept for spatially distributed systems. It can be defined according to open-loop specifications (e.g. the spatial $\mathcal{H}_{2}$ norm [10], [11]), or closedloop specifications (e.g. LQG metrics [14]). We investigate the following definition of modal observability for the $n^{\text {th }}$ eigenmode at location $\left(x_{p}, y_{p}\right)$,

$$
\mathcal{M}\left(n, x_{p}, y_{p}\right):=\frac{\left|K_{n} Z_{n}\left(x_{p}, y_{p}\right)\right|}{(x, y) \in \Omega\left|K_{n} Z_{n}(x, y)\right|},
$$

where $\mathcal{M}: \mathbb{N} \times\left[-L_{x}, L_{x}\right] \times\left[-L_{y}, L_{y}\right] \rightarrow \mathbb{R}$. Equation (25) can be simplified to

$$
\mathcal{M}\left(n, x_{p}, y_{p}\right)=\left|Z_{n}\left(x_{p}, y_{p}\right)\right| .
$$

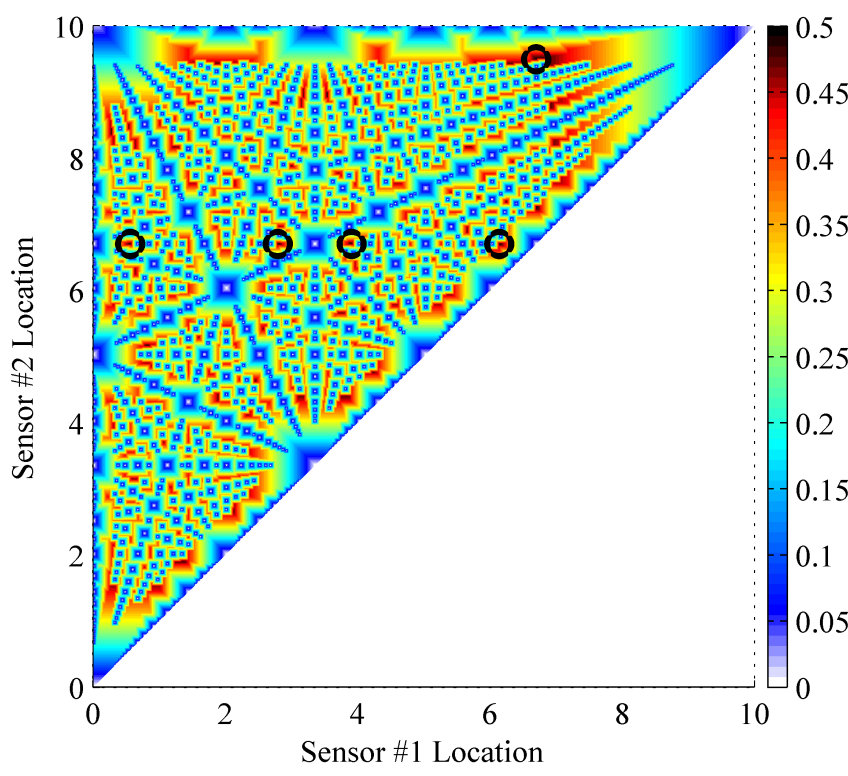

Fig. 2. Sample visualization of $\mathcal{S}_{x}\left(\boldsymbol{x}_{s}\right)$ for two sensors. The global optima are denoted by black circles.

using (18) and (21). Note that $\mathcal{M}\left(n, x_{p}, y_{p}\right) \in[0,1]$, where $\mathcal{M}\left(n, x_{p}, y_{p}\right)=0$ signifies no observability and $\mathcal{M}\left(n, x_{p}, y_{p}\right)=1$ signifies best case observability. Conceptually, the model observability for an eigenmode at a particular spatial location is given by the magnitude of the corresponding eigenfunction. This definition is novel, and is motivated by the idea that one should place sensors where the eigenfunction magnitude is large.

\section{B. Optimization scheme}

1) Separation and Symmetry: The structure of the modal model contains the separation property, which consequently reduces the complexity of the optimization. We decompose the mode shapes of the $N$ eigenmodes into their constituting eigenfunctions $Z_{n}(x, y)=X_{i}(x) Y_{j}(y)$ and from this point on, we work on the $x$ dimension only. The procedure for the $y$ dimension involves identical steps. At the end, the results are recombined. The modal observability measure (26) also separates in two parts

$$
\mathcal{M}_{x}\left(i, x_{p}\right):=\left|X_{i}\left(x_{p}\right)\right| \text { and } \mathcal{M}_{y}\left(j, y_{p}\right):=\left|Y_{j}\left(y_{p}\right)\right| \text {, }
$$

since $\mathcal{M}\left(n, x_{p}, y_{p}\right)=\mathcal{M}_{x}\left(i, x_{p}\right) \mathcal{M}_{y}\left(j, y_{p}\right)$.

Additionally, we reduce the optimization to the positive half of the $x$ subdomain (the interval $\left(0, L_{x}\right)$ ) to avoid multiple isomorphic solutions since the mode shapes are symmetric with respect to the origin, in terms of modal observability.

2) Objective Function Analysis: The nonlinear program, described in Section III-B.4, optimizes the set, $\boldsymbol{x}_{s}$, of sensor locations on the continuous interval $\left(0, L_{x}\right)$ with respect to maximizing the lowest modal observability over all eigenfunctions. Mathematically, the objective function is defined as

$$
\mathcal{S}_{x}\left(\boldsymbol{x}_{s}\right)=\min _{i \in\{1, \ldots, N\}}\left\{\max _{x_{p} \in \boldsymbol{x}_{s}} \mathcal{M}_{x}\left(i, x_{p}\right)\right\} .
$$


Conceptually, each mode is assigned the sensor which is located at the greatest eigenfunction magnitude. Then the objective function value, $\mathcal{S}_{x}\left(\boldsymbol{x}_{s}\right)$, gives us the lowest model observability over the set of modes. Our goal is to maximize the worst case modal observability. Figure 2 provides a sample visualization of $\mathcal{S}_{x}$ for two sensors in the $x$ subdomain. This problem is riddled with multiple local optima. Multiple global optima exist (denoted by circles), and we wish to identify these sensor locations - for two or more sensors. This motivates the following method for selecting optimization starting points. First, we seek to determine the minimal number of sensors required to achieve a given level of observability. Second, we optimize the location of this minimal set of sensors.

3) Minimal Number of Sensors: The first part is formulated as a combinatorial problem. Thus, we establish a binary integer approximation by imposing a grid of $Q$ points on $\left(0, L_{x}\right)$. For every eigenfunction $X_{i}$, we define a binary observability criterion for a hypothetical sensor at the gridpoint $x_{q}, q \in\{1, \ldots, Q\}$ with respect to a modal observability threshold $\tau_{x}^{\text {int }}$ :

$$
\mathcal{B}_{x}(i, q):=\left\{\begin{array}{ll}
1 & \text { for } \mathcal{M}_{x}\left(i, x_{q}\right) \geq \tau_{x}^{i n t} \\
0 & \text { else }
\end{array} .\right.
$$

The value for $\tau_{x}^{i n t}$ is chosen a priori. Since this value depends on the structure of the involved eigenfunctions, it must be chosen appropriately.

The grid structure requires careful consideration to trade off accuracy with complexity. Specifically, the eigenfunction with the greatest spatial frequency provides the appropriate indicator for the grid dimensions. It has feasible regions where its observability is above the threshold $\tau_{x}^{i n t}$. We use an equal-distanced grid with a distance small enough to cover each of its feasible regions by at least two grid points. This is a rather conservative choice, but in our tests turned out to be a good balance between coverage and problem size. One reason is that the optimal sensor locations tend to be at the boundaries of the feasible regions. With this setting, there is at least one grid point less than half of the region away from every boundary for all eigenfunctions.

The binary observability information can be organized in matrix form

$$
\mathcal{B}=\left[\mathcal{B}_{x}(i, q)\right]_{i, q} \quad i=1, \ldots, N \quad q=1, \ldots, Q,
$$

and this matrix is the input to the search algorithm.

Our immediate goal is to select a set of sensor locations with minimal cardinality, such that for each mode $i$, there exists at least one sensor location $q$ in this set where $\mathcal{B}_{x}(i, q)=1$. The brute-force method to solve this problem is full enumeration. This method would find a complete set (i.e. a set of sensors covering all eigenfunctions), establishing the minimal number of sensors. Since there are likely multiple locations that satisfy the minimum observability criterion, all combinations must be evaluated. However, this method is computationally expensive and does not take advantage of the problem's submodularity, a critical property for our method.
TABLE I

COMPARISON OF ITERATIONS PERFORMED BY FULL ENUMERATION AND Modified GREedy ALgorithm

\begin{tabular}{c|c|c|c}
\hline \hline$\tau_{x}^{\text {int }}$ & Full Enumeration & Modified Greedy & Reduction Factor \\
\hline \hline 0.30 & 820 & 245 & 3.3 \\
0.65 & 32567 & 1796 & 18.1 \\
0.82 & 4912381 & 15843 & 310 \\
\hline \hline
\end{tabular}

A problem is submodular, if it has a diminishing return property. In our case we choose one grid point after another to build a set of sensor locations. Each grid point $x_{q}, q \in$ $1, \ldots, Q$ satisfies (29) for a fixed number of eigenfunctions that it can contribute to the set of covered eigenfuntions. The later we add a grid point to a sensor location set, the less additional eigenfunctions it will contribute. This is because more and more eigenfunctions are already covered by previously chosen sensor locations.

Based on submodularity, we have designed a modified greedy algorithm (for details please refer to [18]) that spans a much smaller tree than the complete combinatorial tree, while still covering all branches of interest. This is crucial for achieving a practical algorithm for placing multiple sensors.

Full enumeration and the modified greedy algorithm both return the minimal number of sensors $P$, and the corresponding sets of grid points that satisfy the binary observability criterion.

4) Sensor Location Optimization: In the second step, the sets of sensors from the first part are used as starting points $\boldsymbol{x}_{s 0}$ for the constrained nonlinear programming problem (NLP) we describe next. The NLP optimizes the sensor locations $\boldsymbol{x}_{s}$ on the continuous interval $\left(0, L_{x}\right)$ with respect to maximizing the lowest modal observability over all eigenfunctions, $\mathcal{S}_{x}\left(\boldsymbol{x}_{s}\right)$ defined in (28). The optimization problem is thus formulated as

$$
\begin{gathered}
\max _{\boldsymbol{x}_{s} \in\left(0, L_{x}\right)^{P}} \mathcal{S}\left(\boldsymbol{x}_{s}\right), \\
\text { s. to } \\
x_{s, 1} \leq x_{s, 2} \leq \cdots \leq x_{s, P},
\end{gathered}
$$

for every set of starting points $\boldsymbol{x}_{s 0}$. Constraint (32) prevents permutations in the sensor order. Note that multiple starting points may converge to identical optima. This optimization problem can be solved with gradient-based algorithms, such as sequential quadratic programming.

\section{EXAmple Results}

To illustrate some results, we assume a battery pack with dimensions defined by $L_{x}=L_{y}=10$. We set the ratio between the thermal conductivity inside the pack material and the heat transfer rate from pack material to surrounding air to

$$
\frac{\lambda}{k_{p}}=20
$$

corresponding to moderate heat conductivity inside the pack in combination with high heat transfer over the boundaries. 
Eigenfunctions (normalized)

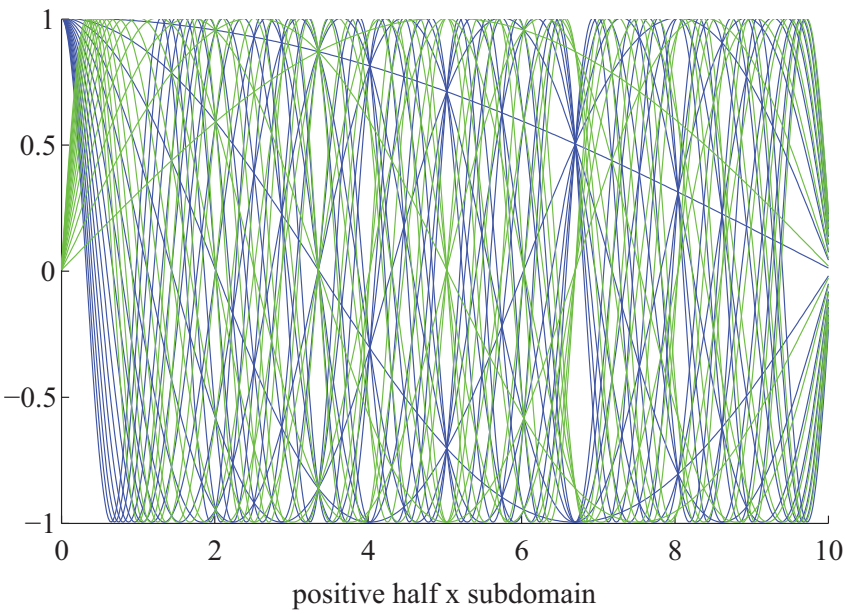

Fig. 3. Example: The first 31 eigenfunctions $X_{i}$, for the $x$ dimension. Green and blue correspond to sine and cosine eigenfunctions, respectively.

(a) Two sensors (objective $=0.50$ )

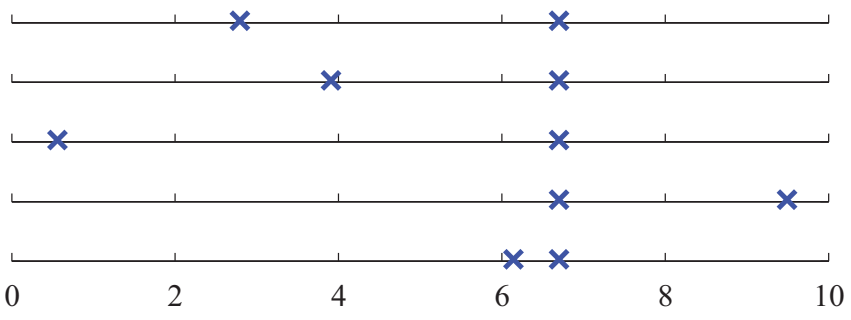

(b) Three sensors (objective $=0.77$ )

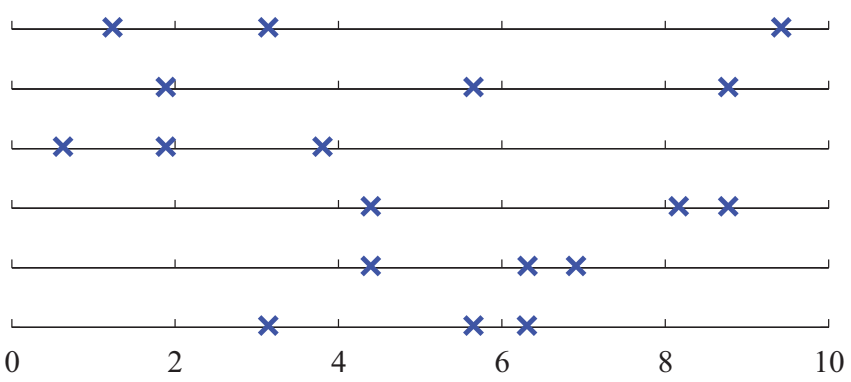

(c.1) Four sensors (objective $=0.88$ )

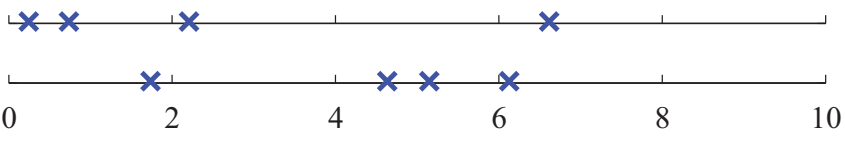

(c.2) Four sensors (objective $=0.87$ )

\begin{tabular}{|c|c|c|c|c|c|}
\hline 0 & 2 & 4 & 6 & 8 & 10 \\
\hline
\end{tabular}

Fig. 4. Optimal locations for (a) two, (b) three, and (c.1) four sensors. For each number of sensors, multiple optima with identical objectives $\mathcal{S}_{x}\left(\boldsymbol{x}_{s}^{*}\right)$ exist. The sensor locations in (c.2) are suboptimal, yet exhibit more equally distanced spacing after reflecting two sensors onto the left-half of the subdomain.
The finite subset of eigenmodes is defined by selecting the bandwidth of interest. Since the eigenvalues decrease quadratically towards $-\infty$, we truncate the high frequency modes and retain the dominant low frequency modes. In this example, 185 eigenmodes are retained. This example set of eigenmodes is decomposed into the eigenfunctions. Figure 3 shows the first 31 eigenfunctions for the $x$ dimension.

Table I shows a comparison of the required iterations for the modified greedy algorithm and full enumeration. In this example, capitalizing on the submodularity property provides a reduction of up to two orders of magnitude.

Figure 4(a)-(c) shows the resulting optimal sensor sets. In each case, multiple sensor sets are shown because multiple equivalent optima were identified. The sets of two sensors in Figure 4(a) where obtained by setting $\tau_{x}^{i n t}=0.3$ for the integer optimization. The maximum observability following the nonlinear optimization is $\mathcal{S}_{x}\left(\boldsymbol{x}_{s}^{*}\right)=0.5$. One may see that the NLP successfully identifies the global optimaIn shown in Fig. 2,

Setting $\tau_{x}^{i n t}=0.65$ requires one additional sensor. The optimal locations for several sets of three sensors are provided in Figure 4(b). These sensor sets achieve $\mathcal{S}_{x}\left(\boldsymbol{x}_{s}^{*}\right)=0.77$. Further increasing the initial $\tau_{x}^{\text {int }}=0.82$ provokes a fourth sensor and increases the observability objective to $\mathcal{S}_{x}\left(\boldsymbol{x}_{s}^{*}\right)=$ 0.88, as shown in Figure 4(c.1).

Note that the total observability for the eigenmodes is $\mathcal{S}\left(\boldsymbol{x}_{s}^{*}, \boldsymbol{y}_{s}^{*}\right)=\mathcal{S}_{x}\left(\boldsymbol{x}_{s}^{*}\right) \mathcal{S}_{y}\left(\boldsymbol{y}_{s}^{*}\right)$. Therefore, $\mathcal{S}\left(\boldsymbol{x}_{s}^{*}, \boldsymbol{y}_{s}^{*}\right)=0.25$ if $\mathcal{S}_{x}\left(\boldsymbol{x}_{s}^{*}\right)=\mathcal{S}_{y}\left(\boldsymbol{y}_{s}^{*}\right)=0.5$, and $\mathcal{S}\left(\boldsymbol{x}_{s}^{*}, \boldsymbol{y}_{s}^{*}\right)=0.76$ for $\mathcal{S}_{x}\left(\boldsymbol{x}_{s}^{*}\right)=\mathcal{S}_{y}\left(\boldsymbol{y}_{s}^{*}\right)=0.87$.

\section{A. Implementation Issues}

Choosing one of the optimal sensor sets from Fig. 4 is based on the requirements of the application. For estimating temperatures in large battery packs, one critical aspect is the worst case time delay between a local failure and a sensor, due to thermal conductivity limits. To minimize this effect, we would prefer sets with the most equally spaced distribution. Another aspect is the way the sensor set is modified to cover the right half of the $x$ subdomain. One choice is to duplicate a sensor set by mirroring at the origin, providing two redundant sensors for each eigenmode while accepting a lower minimal modal observability. For example, a total of four sensors would be achieved by mirroring the first location set from Figure 4(a). The other option is to take a sensor set from the results with more sensors and a higher minimal modal observability and completely move half of the sensors to corresponding locations on the other half of the subdomain. Figure 4(c.2) illustrates such an example. This set has a slightly lower minimal modal observability ( 0.87 vs. $\left.\mathcal{S}_{x}\left(\boldsymbol{x}_{s}^{*}\right)=0.88\right)$, but is the most equally distanced set for the case of moving two sensors to the left half of the $x$ subdomain. The key difference is the former design choice provides sensor redundancy whereas the latter provides higher minimal modal observability.

\section{CONClusion}

This paper examines sensor placement for estimation of battery pack thermal dynamics. Specifically, we seek the 
location of a minimal set of sensors that optimize a particular observability criterion. The critical ideas can be summarized into four points. First, we derive a finite-dimensional modal model via eigenanalysis. Second, we quantify the observability of an eigenmode at a given spatial location by the eigenmode's value. Third, we determine the minimal set of sensors which all satisfy a particular observability criterion. This procedure capitalizes on the submodularity property of the optimization problem. Finally, we optimize the locations of this minimal sensor set via a nonlinear program. This paper provides a systematic methodology for placing temperature sensors in large battery packs for estimating thermal dynamics.

\section{ACKNOWLEDGMENT}

P. Wolf thanks Prof. O. Sawodny for providing support during his tenure as a visiting scholar at UC San Diego.

\section{REFERENCES}

[1] K. Smith and C.-Y. Wang, "Power and thermal characterization of a lithium-ion battery pack for hybrid-electric vehicles," Journal of Power Sources, vol. 160, no. 1, pp. 662 - 673, 2006.

[2] S. J. Moura, N. Chaturvedi, and M. Krstic, "PDE Estimation Techniques for Advanced Battery Management Systems - Part I: SOC Estimation," in Proceedings of the 2012 American Control Conference, Montreal, Canada, June 2012.

[3] — - "PDE Estimation Techniques for Advanced Battery Management Systems - Part II: SOH Identification," in Proceedings of the 2012 American Control Conference, Montreal, Canada, June 2012.

[4] S. J. Moura, J. L. Stein, and H. K. Fathy, "Battery Health-Conscious Power Management for Plug-in Hybrid Electric Vehicles via Stochastic Control," vol. 1, Cambridge, MA, United states, 2010, pp. 615 - 624 .

[5] C. Park and A. Jaura, "Dynamic thermal model of li-ion battery for predictive behavior in hybrid and fuel cell vehicles," SAE transactions, vol. 112 , no. 3 , pp. 1835-1842, 2003.
[6] M. Guo and R. E. White, "Thermal model for lithium ion battery pack with mixed parallel and series configuration," Journal of the Electrochemical Society, vol. 158, no. 10, pp. A1166 - A1176, 2011.

[7] X. Hu, S. Lin, S. Stanton, and W. Lian, "A state space thermal model for hev/ev battery modeling," SAE 2011 World Congress and Exhibition, 2011.

[8] H. Sun, X. Wang, B. Tossan, and R. Dixon, "Three-dimensional thermal modeling of a lithium-ion battery pack," Journal of Power Sources, 2012.

[9] D. Uciński, Optimal measurement methods for distributed parameter system identification. CRC, 2005.

[10] D. Halim and S. Reza Moheimani, "An optimization approach to optimal placement of collocated piezoelectric actuators and sensors on a thin plate,' Mechatronics, vol. 13, no. 1, pp. $27-47,2003$.

[11] A. Armaou and M. A. Demetriou, "Optimal actuator/sensor placement for linear parabolic PDEs using spatial H2 norm," Chemical Engineering Science, vol. 61, no. 22, pp. 7351 - 7367, 2006.

[12] Y. Lou and P. D. Christofides, "Optimal actuator/sensor placement for nonlinear control of the kuramoto-sivashinsky equation," IEEE Transactions on Control Systems Technology, vol. 11, no. 5, pp. 737 $-745,2003$.

[13] K. K. Chen and C. W. Rowley, "H2 optimal actuator and sensor placement in the linearised complex Ginzburg-Landau system," Journal of Fluid Mechanics, vol. 681, pp. 241 - 260, 2011.

[14] J. Borggaard, J. Burns, and L. Zietsman, "On using lqg performance metrics for sensor placement," in Proc. American Control Conf. (ACC), 2011, pp. 2381-2386.

[15] C. Kubrusly and H. Malebranche, "Sensors and controllers location in distributed systems-a survey," Automatica, vol. 21, no. 2, pp. 117-128, 1985.

[16] A. Smyshlyaev, M. Krstic, N. Chaturvedi, J. Ahmed, and A. Kojic, "PDE Model for Thermal Dynamics of a Large Li-ion Battery Pack," in Proc. American Control Conf. (ACC), 2011, pp. 959-964.

[17] A. Krause, J. Leskovec, C. Guestrin, J. VanBriesen, and C. Faloutsos, "Efficient sensor placement optimization for securing large water distribution networks," Journal of Water Resources Planning and Management, vol. 134, no. 6, pp. 516 - 526, 2008.

[18] P. Wolf, "Spatio-temporal temperature estimation for large battery packs," Diploma Thesis, Institute for System Dynamics, University of Stuttgart, 2012.

[19] G. Cain and G. Meyer, Separation of variables for partial differential equations: an eigenfunction approach. CRC Press, 2006. 